\title{
A single-center retrospective analysis of childhood hepatoblastoma in China
}

\author{
Wenya Yu ${ }^{1 \#}$, Xiang Liu ${ }^{2 \#}$, Jingquan Li $^{1 \#}$, Zhifeng Xi ${ }^{3}$, Jing Jin ${ }^{3}$, Hongting Huang ${ }^{3}$, Yang Ge ${ }^{1}$, Qiang Xia ${ }^{3}$ \\ ${ }^{1}$ School of Public Health, Shanghai Jiao Tong University School of Medicine, Shanghai, China; ${ }^{2}$ Department of Respiratory Disease, The 903 rd \\ Hospital of PLA, Hangzhou, China; ${ }^{3}$ Department of Liver Surgery, Renji Hospital, Shanghai Jiao Tong University School of Medicine, Shanghai, \\ China \\ Contributions: (I) Conception and design: W Yu, X Liu, Y Ge, Q Xia; (II) Administrative support: J Li, Y Ge, Q Xia; (III) Provision of study materials \\ or patients: Z Xi, J Jin, H Huang, Q Xia; (IV) Collection and assembly of data: Z Xi, J Jin, H Huang; (V) Data analysis and interpretation: W Yu, X \\ Liu, J Li, Y Ge; (VI) Manuscript writing: All authors; (VII) Final approval of the manuscript: All authors. \\ \#These authors contributed equally to this work. \\ Correspondence to: Qiang Xia. Department of Liver Surgery, Renji Hospital, Shanghai Jiao Tong University School of Medicine, Shanghai, \\ China. Email: xiaqiang@shsmu.edu.cn; Yang Ge. School of Public Health, Shanghai Jiao Tong University School of Medicine, Shanghai, China. \\ Email: geyang19861026@icloud.com.
}

\begin{abstract}
Background: This study aimed to investigate the critical factors associated with prognosis for children with hepatoblastoma (HB) in mainland China combined with the aspect of health economics and management.

Methods: This study retrospectively reviewed children with HB in Renji Hospital Affiliated to the Shanghai Jiao Tong University School of Medicine from January 2013 to December 2019. Descriptive analysis was used to describe the essential characteristics. Kaplan-Meier method and Cox proportional hazard models were used to estimate the survival rate and prognosis factors.

Results: For the 87 children with HB, the average survival was 2,002.8 days (95\% CI: 1,798.7-2,206.9 days), the 1 - and 5 -year survival rates were $87.7 \%$ and $78.9 \%$, respectively. Undergoing surgery and sex were independent prognostic factors of childhood HB. Children with HB undergoing hepatectomy (HR: 0.039) or liver transplantation (HR: 0.142) had a better prognosis, while boys were associated with a poorer prognosis (HR: 3.614). The average medical expenses for childhood HB were 40,217.5 $\pm 3,862.0 \mathrm{CNY}$ and liver transplantation cost more than hepatectomy.

Conclusions: The results had a comparable survival rate with other studies globally. Surgical therapy and sex are associated with the prognosis of children with HB. The economic burden of childhood HB deserves to be further explored.
\end{abstract}

Keywords: Hepatoblastoma (HB); children; prognosis; survival

Submitted Jul 24, 2020. Accepted for publication Sep 30, 2020.

doi: $10.21037 /$ gs-20-710

View this article at: http://dx.doi.org/10.21037/gs-20-710

\section{Introduction}

Primary liver cancer is a rare disease in children globally, accounting for $1-2 \%$ of all pediatric cancers (1-3). The most common primary liver cancer is hepatoblastoma (HB), which makes up approximately 50-80\% (3-10), and with a much higher proportion (91\%) among children under 5 years old $(8,11,12)$.
The incidence rate of pediatric HB varies in different countries and regions and is more common in infants (4). The incidence rate of HB in the United States (4), in Europe (13), and South Africa (14) was 0.8-1.5, 1.2, and 0.61 per million children, respectively. For survival, the survival rate of $\mathrm{HB}$ has passed $70 \%$ (15-17). A study in the US showed the 1-, 5-, and 10-year survival for HB was $81.5 \%, 71.6 \%$, and $69 \%$, respectively (18). Another 
study in Europe found that the 5-year survival rate for $\mathrm{HB}$ was $66 \%$ (13). It was higher for patients undergoing liver transplant or resection. The 1-, 5-, and 10-year survival rate for $\mathrm{HB}$ was $93 \%, 82 \%$, and $82 \%$, respectively (19).

However, compared with studies in epidemiology, primary, and clinical medicine globally, there are only a few studies in China, especially in mainland China. A multicenter study on $153 \mathrm{HB}$ children reported that the 6-year survival rate was $83.3 \% \pm 3.1 \%(15)$. However, results of single-center studies varied. A study in Shanghai on 104 children with $\mathrm{HB}$ reported the 5-year survival rate of $86.3 \pm 5.0 \%$ (20). A study in Beijing on 176 children with $\mathrm{HB}$ reported that the 5 -year survival rate was $54.6 \%$ (21). Another study in Beijing on 102 children with HB found that advanced $\mathrm{HB}$ was associated with a low survival rate, from $75.0 \%$ in stage II to $20.2 \%$ in stage IV (22). In addition, studies in Hong Kong and Taiwan always concentrated on the comparisons between HB and hepatocellular carcinoma $(23,24)$.

Therefore, considering the scarcity of studies in China, this study aimed to describe the epidemiological characteristics and analyze the influencing factors of survival among children with HB in mainland China, which would provide some evidence for the improvement of prognosis for children with HB. We present the following article in accordance with the STROBE reporting checklist (available at http://dx. doi. org/10.21037/gs-20-710).

\section{Methods}

\section{Inclusion and exclusion criteria}

This study retrospectively reviewed 115 children with HB treated in Renji Hospital Affiliated to the Shanghai Jiao Tong University School of Medicine, Shanghai, China, from January 2013 to December 2019. However, 87 patients were enrolled in this study. The enrollment criteria in this study were: (I) patients should be younger than 18 years old; (II) patients should be dragonized as HB; (III) patients or their parents should participate in this study. The exclusion criteria were: (I) patients or their parents refused to be followed up; (II) patients did not accept any treatment.

The Ethics Committee approved the study protocol of the Shanghai Jiao Tong University School of Medicine. The written informed consent of all enrolled patients or their parents was obtained. Patient anonymity was guaranteed to be preserved. The study was conducted in accordance with the Declaration of Helsinki (as revised in 2013).

\section{Diagnosis and treatment}

The diagnosis was on clinical manifestations, laboratory examinations, and pathological examinations. Patients were treated with hepatectomy, liver transplantation, or without surgery on their conditions. Most of the patients not undergoing surgery were hospitalized for biopsy and clinical evaluations and did not experience further surgical treatment.

\section{Follow-up}

The last follow-up date was December 31, 2019. Follow-ups were conducted after patients discharged from the hospital. Overall survival (OS) was estimated as time (in days) from diagnosis to death of any cause. Patients not experiencing an event were censored from the last contact.

\section{Statistical analysis}

Two researchers entered all the data simultaneously. Data collection and analysis were performed using SPSS 18.0 (Statistical Package for the Social Science, SPSS Inc., Chicago, IL, USA). Descriptive analysis was used to describe the demographic and disease characteristics. Then, the continuous variables were described as mean \pm SEM. Categorical variables are described by number and percentage. Survival time, survival rates, and survival curves were estimated by the Kaplan-Meier method. A log-rank test was used in the univariate analysis. Variables statistically significant in the univariate analysis, and variables with professional meanings on previous publications, were included in the Cox proportional hazards model. All tests were two-way, and $\mathrm{P}<0.05$ was considered statistically significant.

\section{Results}

\section{Patients' characteristics}

Eighty-seven children were recruited into this study from Shanghai Renji Hospital. The primary characteristics of the patients were summarized in Table 1. The sex distribution was $1: 1.23(\mathrm{M}: \mathrm{F})$. The average age at diagnosis was $3.0 \pm 0.3$ years old (range, 0.0 to 12.0 years old), of which the majority $(81.6 \%)$ were under 5 years old. More than half $(64.4 \%)$ of patients underwent hepatectomy, $19.5 \%$ had liver transplantation, and $16.1 \%$ did not experience any surgery. Most patients (88.5\%) underwent chemotherapy 
Table 1 Demographic and clinical characteristics $(\mathrm{N}=87)$

\begin{tabular}{|c|c|c|}
\hline Characteristic & $\begin{array}{c}\text { No. of } \\
\text { patients/mean }\end{array}$ & $\% / S E M$ \\
\hline \multicolumn{3}{|l|}{ Sex } \\
\hline Male & 39 & 44.8 \\
\hline Female & 48 & 55.2 \\
\hline Age at diagnosis & 3.0 & 0.3 \\
\hline$\leq 5$ years old & 71 & 81.6 \\
\hline$>5$ years old & 16 & 18.4 \\
\hline \multicolumn{3}{|l|}{ Surgery } \\
\hline Hepatectomy & 56 & 64.4 \\
\hline Liver transplantation & 17 & 19.5 \\
\hline No surgery & 14 & 16.1 \\
\hline \multicolumn{3}{|l|}{ Preoperative chemotherapy } \\
\hline Yes & 77 & 88.5 \\
\hline No & 10 & 11.5 \\
\hline \multicolumn{3}{|l|}{ Postoperative chemotherapy } \\
\hline Yes & 72 & 82.8 \\
\hline No & 15 & 17.2 \\
\hline Length of stay (days) & 16.3 & 1.1 \\
\hline $0-3$ & 5 & 5.7 \\
\hline $4-7$ & 9 & 10.3 \\
\hline $8-10$ & 13 & 14.9 \\
\hline $11-15$ & 23 & 26.4 \\
\hline$\geq 16$ & 37 & 42.5 \\
\hline \multicolumn{3}{|l|}{ Presenting sign } \\
\hline Abdominal distension/abdominal mass & 32 & 36.8 \\
\hline Increased abdominal circumference & 12 & 13.8 \\
\hline Abdominal pain and discomfort & 7 & 8.0 \\
\hline Fever/recurrent fever & 8 & 9.2 \\
\hline Asymptomatic medical examination & 15 & 17.2 \\
\hline Loss of appetite/nausea and vomiting & 5 & 5.7 \\
\hline Abnormal urine & 3 & 3.4 \\
\hline Yellow staining of skin sclera & 2 & 2.3 \\
\hline Cough & 1 & 1.1 \\
\hline Abdominal pain after trauma & 2 & 2.3 \\
\hline Others & 12 & 13.8 \\
\hline Medical expenses $\left(\mathrm{CNY}^{\dagger}\right)$ & $40,217.5$ & $3,862.0$ \\
\hline
\end{tabular}

${ }^{\dagger} \mathrm{CNY}$ : Chinese Yuan (currency unit). before surgery, including those without surgery, while fewer patients $(82.8 \%)$ underwent chemotherapy after surgery. The average lengths of stay were $16.3 \pm 1.1$ days (range, 1 to 55 days), and a half ( $42.5 \%)$ stayed in the hospital at least 16 days. The most frequent presenting sign was abdominal distension or abdominal mass (36.8\%). The average medical expenses were 40,217.5 $\pm 3,862.0 \mathrm{CNY}$ (Chinese Yuan; $\sim \mathrm{US} \$ 5,745.4 \pm \mathrm{US} \$ 551.7)$.

\section{Survival descriptions of different subgroups}

For all 87 patients, the overall average survival was 2,002.8 days (95\% CI: 1,798.7-2,206.9 days), the 1-year OS and the 5 -year OS were $87.7 \%$ and $78.9 \%$, respectively (Table 2 and Figure 1A). Descriptive survival statistics and Kaplan-Meier curves showed that surgery and chemotherapy after surgery had prognostic significance. The 1-year OS rates of boys and girls were $86.5 \%$ and $88.7 \%(\mathrm{P}=0.485)$, respectively; the 5 -year OS rates were $67.3 \%$ and $85.6 \%$, respectively (Table 2 and Figure $1 B$ ). The 1 -year OS for patients under 5 years old was $86.5 \%$ and $92.9 \%$ in patients over 5 years old (Table 2 and Figure 1C, $\mathrm{P}=0.852$ ). Undergoing hepatectomy was associated with an increasing average survival from 753.1 to $2,248.9$ days and a 1-year OS rate from $42.9 \%$ to $95.7 \%$; liver transplantation was associated with an increasing 1-year OS rate from $42.9 \%$ to $90.0 \%$ (Table 2 and Figure $1 D, \mathrm{P}<0.0001$ ). The 1 -year OS rate for patients with preoperative chemotherapy was $88.6 \%$, and $80.0 \%$ for those without (Table 2 and Figure $1 E, \mathrm{P}=0.965)$. Patients having postoperative chemotherapy are associated with an increasing 1-year OS rate from $66.7 \%$ to $91.9 \%$ (Table 2 and Figure $1 F, \mathrm{P}=0.007$ ). The 1-year OS rates for patients staying in hospital for $0-3$, $4-7,8-10,11-15$, and $\geq 16$ days were $60.0 \%, 64.8 \%, 92.3 \%$, $90.9 \%$, and $93.3 \%$, respectively (Table 2 and Figure $1 G$, $\mathrm{P}=0.100)$. The average survival rates, the 1 - and 5 -year OS, for patients with various presenting signs were shown in Table 2 and Figure 2 (all $\mathrm{P}$ values $>0.05$ ).

\section{Cox proportional hazard analysis}

Factors with statistical significance in the univariate analysis were included in the Cox proportional hazard model. Sex was chosen on existing studies (15). The multivariate analysis suggested that undergoing surgery and sex were independent prognostic factors of childhood HB (Table 3, $\mathrm{P}<0.05)$. Undergoing hepatectomy (HR: 0.039, Figure $3 A$ ) 
Table 2 Descriptive survival statistics

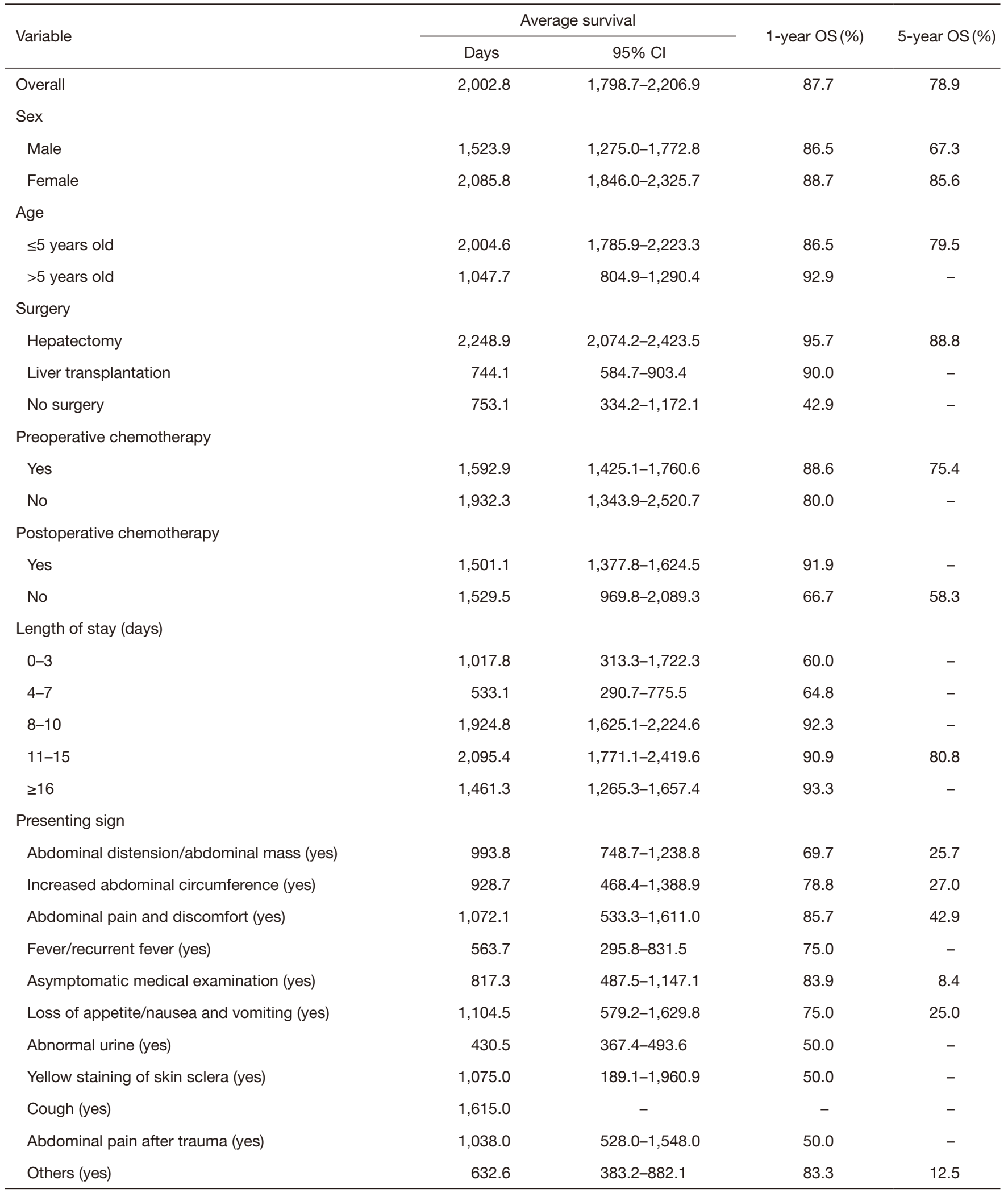

$\mathrm{Cl}$, confidence interval; OS, overall survival. 

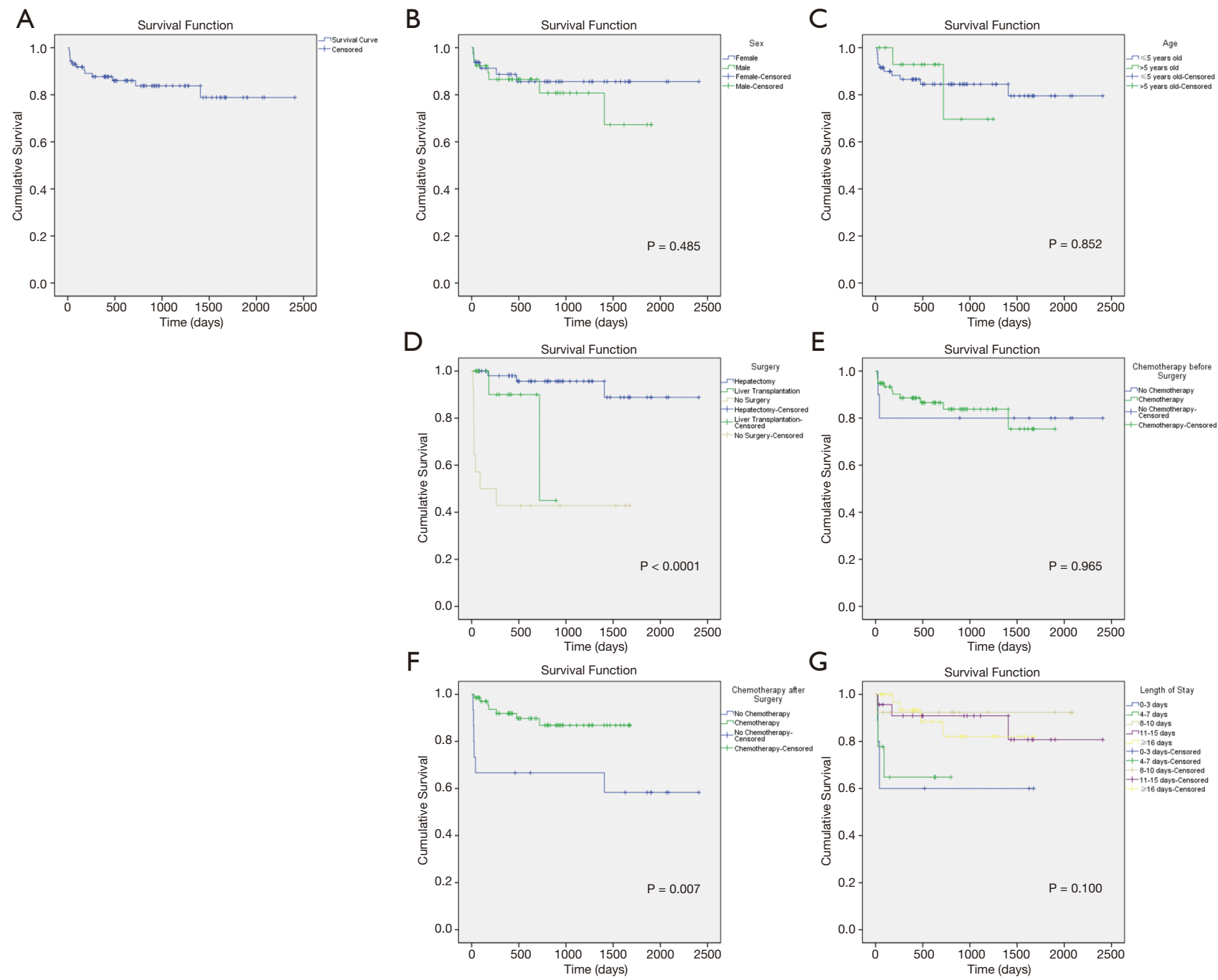

Figure 1 Overall survival estimates. (A) The overall survival curve; (B) stratified by sex $(\mathrm{P}=0.485)$; (C) stratified by age (P=0.852); (D) stratified by surgery $(\mathrm{P}<0.0001)$; (E) stratified by preoperative chemotherapy $(\mathrm{P}=0.965)$; $(\mathrm{F})$ stratified by postoperative chemotherapy $(\mathrm{P}=0.007) ;(\mathrm{G})$ stratified by length of stay $(\mathrm{P}=0.100)$.

or liver transplantation (HR: 0.142 , Figure $3 A$ ) was associated with a better prognosis, while boys were associated with a poorer prognosis (HR: 3.614, Figure 3B).

\section{Comparison of the medical expenses of different surgical approaches}

Surgery could lead to a better prognosis, and patients with hepatectomy had better outcomes than those with liver transplantation. Therefore, it would be meaningful for parents and doctors to consider more about medical expenses when determining the treatment for children with HB because the economic burden was always an essential factor impeding the treatment effect. For patients undergoing hepatectomy, their average medical expenses

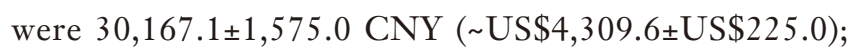
for those undergoing liver transplantation, their average medical expenses were 99,633.9 $99,154.3 \mathrm{CNY}$ ( US\$14,233.4 \pm US\$1,307.8); for those not having surgery, their average medical expenses were $8,270.7 \pm 2,566.2 \mathrm{CNY}$ ( US\$1,181.5 \pm US\$366.6). Also, because the Levene test showed the uneven variance $(\mathrm{P}<0.0001)$, the Kruskal-Wallis $\mathrm{H}$ test was used to explore the difference between the medical expenses of the three groups. Statistics suggested 

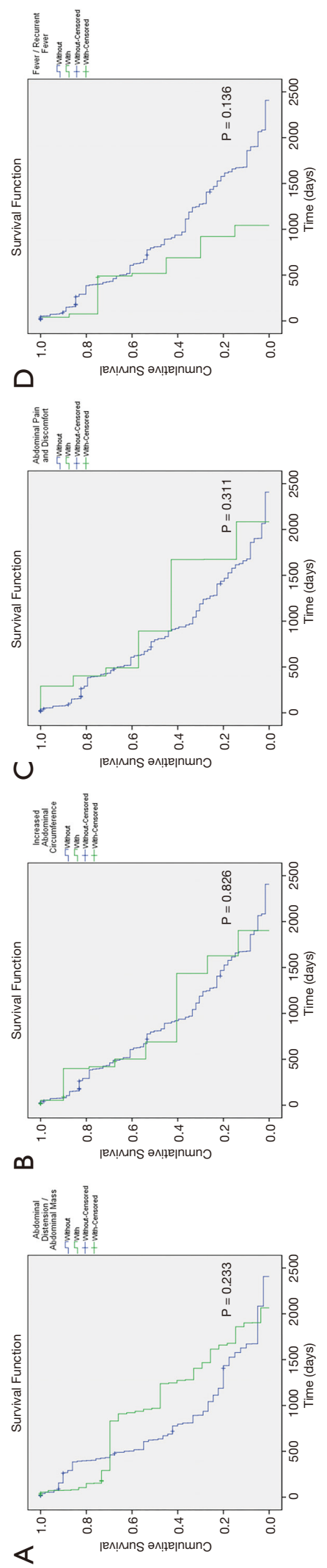

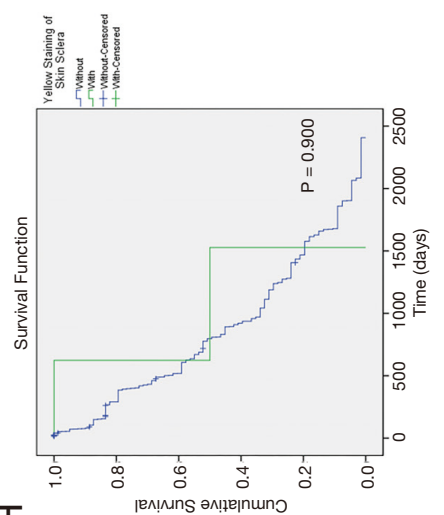

I
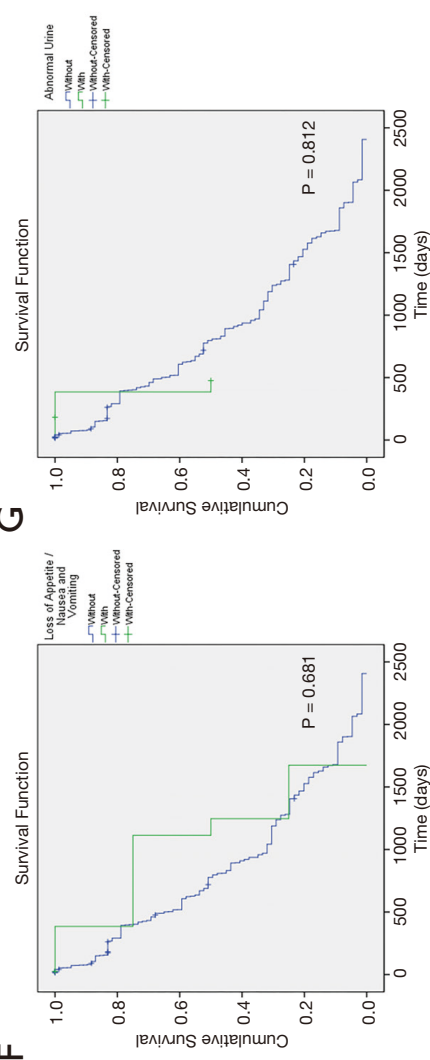

丩

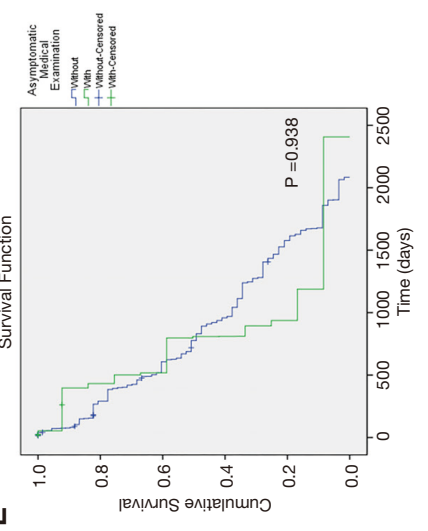

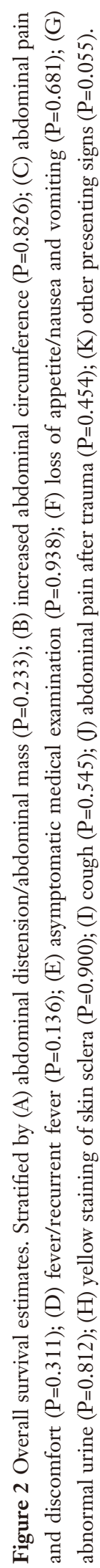
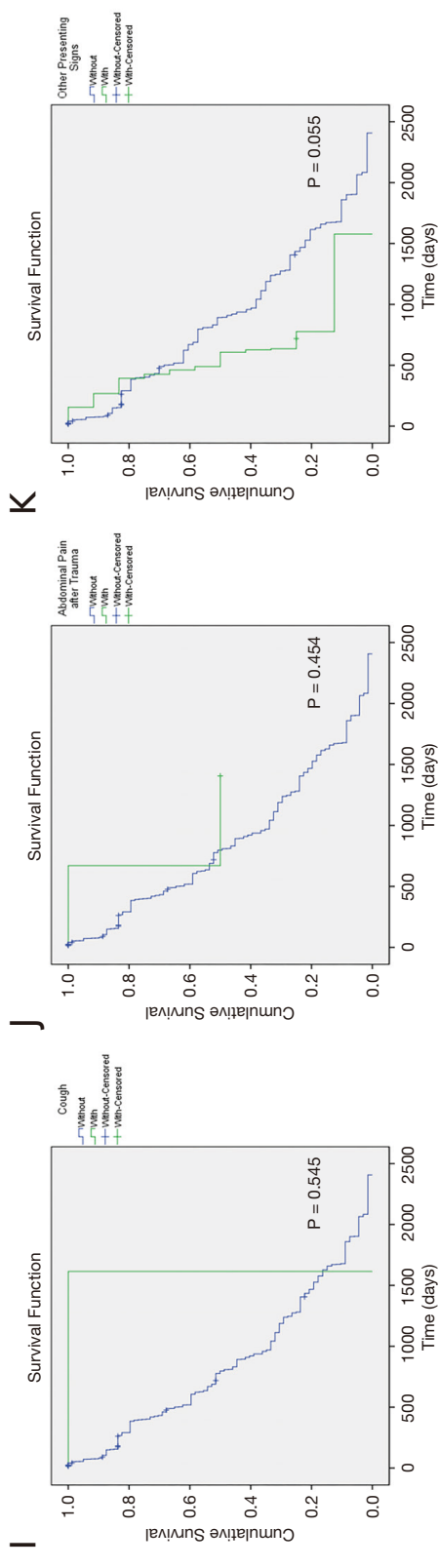
that the medical expenses of the three groups were statistically different $(\mathrm{P}<0.0001)$. The Mann-Whitney $\mathrm{U}$ test showed that the medical expenses between hepatectomy and liver transplantation $(\mathrm{P}<0.0001)$, hepatectomy, and no surgery $(\mathrm{P}<0.0001)$, liver transplantation, and no surgery $(\mathrm{P}<0.0001)$ were all statistically significant (Table 4).

Table 3 Cox proportional hazard analysis

\begin{tabular}{lccc}
\hline Variable & HR & $95 \% \mathrm{Cl}$ & $\mathrm{P}$ value \\
\hline Surgery & & & \\
Hepatectomy & 0.039 & $0.009-0.162$ & $<0.0001$ \\
Liver transplantation & 0.142 & $0.028-0.716$ & 0.018 \\
No surgery & ref & ref & ref \\
Sex & & & \\
Male & 3.614 & $1.075-12.152$ & 0.038 \\
Female & ref & ref & ref \\
$\begin{array}{l}\text { Postoperative } \\
\text { chemotherapy }\end{array}$ & & & \\
Yes & - & - & 0.296 \\
No & ref & ref & ref \\
\hline
\end{tabular}

$\mathrm{HR}$, hazard ratio; $\mathrm{Cl}$, confidence interval; ref, reference.

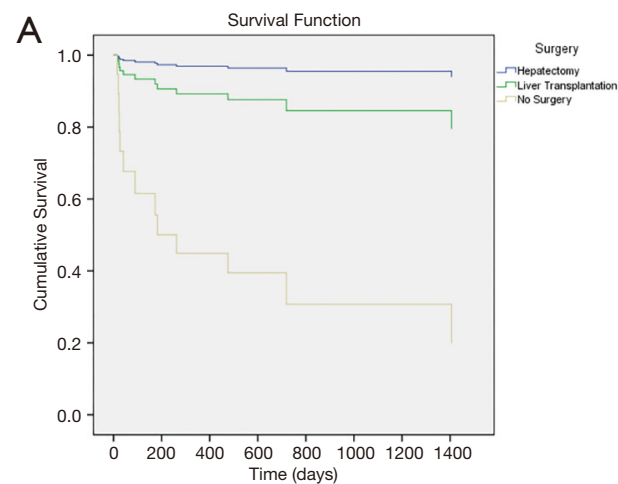

\section{Discussion}

In the past four decades, the diagnosis and treatment of pediatric HB have developed a lot globally $(25,26)$, which led to a clear increase in the survival rate of children with HB. Statistics in the 1980s showed that the 5 -year survival rate for children with $\mathrm{HB}$ was $36 \%$ (27). However, combined with modern chemotherapy and surgical technologies $(16,28,29)$, the 5 -year survival rate in the United States from 1983 to 2005 was $76 \%$ (27), and in Spain, the 5-year survival rate from 1991 to 2009 was $73.2 \%$ (30). Consistent with global trends, this study in China from 2013 to 2019 showed that the 5-year OS was $78.9 \%$, which was comparable with other single-center studies nationwide. A single-center study in 2014 in Beijing suggested that the 5 -year OS was $66.7 \%$ (21). However, the 5 -year OS in another single-center study in Shanghai from 2011 to 2017 was $86.3 \%$ (20). It was like the 5 -year OS of 77.7\% from a Hong Kong study from 1996 to 2014 (31).

To explore the factors affecting the prognosis of childhood HB, the results suggested that undergoing surgery, including hepatectomy and liver transplantation, was associated with a better prognosis. Earlier studies have dug a lot in hepatectomy and liver transplantation. Surgical therapy plays a significant role in improving prognosis,

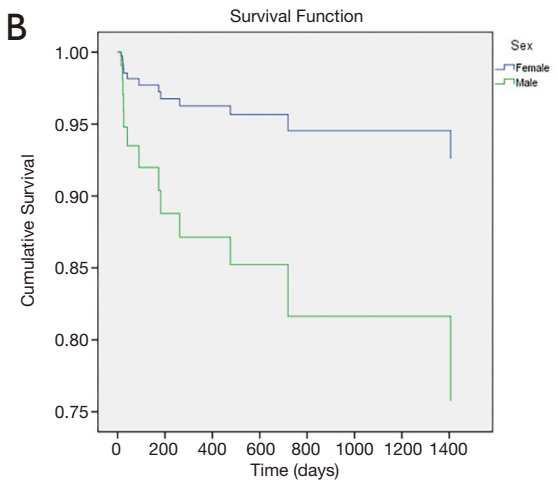

Figure 3 The survival curve. (A) Stratified by surgery after controlling other factors; (B) stratified by sex after controlling other factors.

Table 4 Difference analysis of medical expenses in different surgical groups

\begin{tabular}{lccccc}
\hline \multirow{2}{*}{ Group } & Mean & SEM & P value: Kruskal-Wallis & \multicolumn{2}{c}{ P value: Mann-Whitney U test } \\
\cline { 4 - 5 } & & & H test & 1 vs. 2 & 1 vs. 3 \\
\hline Hepatectomy [1] & $30,167.1$ & $1,575.0$ & $<0.0001$ & $<0.0001$ \\
Liver transplantation [2] & $99,633.9$ & $9,154.3$ & & $<0.0001$ \\
No surgery [3] & $8,270.7$ & $2,566.2$ & & $<0.0001$ \\
\hline
\end{tabular}


especially hepatectomy, combined with chemotherapy. Although over $60 \%$ of tumors appear unresectable at the beginning (16), approximately $40 \%$ can be resected after chemotherapy (32-34). For the left $20 \%$ of tumors, liver transplantation is the best solution (35), which provides a potential cure opportunity for children with advanced HB (36,37). Both surgery therapies improved the survival rate for children with $\mathrm{HB}$ in various stages. The results showed that the 1- and 5-year OS rates for children with hepatectomy were $95.7 \%$ and $88.8 \%$, respectively, and the 1-year OS for children with liver transplantation was $90.0 \%$, which was following previous research. Several studies in the 1990s showed the long-term survival rates for children with hepatectomy varied from $63 \%$ to 94\% (38-41); another study from 1990 to 2013 in the Netherlands suggested children undergoing hepatectomy had the 5 -year survival of $92 \%$ (42). For children undergoing liver transplantation, the 1-year survival rates in the United States varied from $79 \%$ to $92 \%(36,37,43)$; the survival rates in different countries ranged from $62.5 \%$ to $90 \%$ (37). Therefore, it was highly recommended that children with HB be treated by hepatectomy or liver transplantation. To further explore the two surgical therapies, their combination with chemotherapy contributed significantly to the improvement of survival (44). Preoperative chemotherapy can help more children be suitable for hepatectomy $(4,37,45)$, and postoperative chemotherapy can dramatically improve the survival rate, whether for hepatectomy or liver transplantation $(32,33)$. Although the results and some other studies suggested whether receiving chemotherapy before or after surgery did not show significant influence on prognosis $(46,47)$, the reason could not be attributed to chemotherapy hastily, and routine chemotherapy should be conducted according to treatment guidelines $(48,49)$. The guidelines also suggest that postoperative chemotherapy can completely or partially relieve intrahepatic metastasis and recurrence of hepatoblastoma caused by postoperative residual. The sample size should be expanded further to testify these effects.

Also, although international studies showed that male children were more sensitive to $\mathrm{HB}$, there was no evidence verifying the influence of sex on survival $(17,50)$. However, considering a Chinese study suggested that a male was an individual factor contributing to poorer prognosis (15), sex was included in the multivariate analysis even though it was not statistically significant in the univariate analysis. Like this previous publication in China, our study showed that sex was an influencing factor of prognosis, and boys had a poorer prognosis than girls. On this finding, it is necessary to explore further the relationship between sex and prognosis of childhood HB. The differences among various countries and regions should be further considered.

Almost no studies ever reported medical expenses of childhood HB. However, it should be noted that the economic burden is always a significant obstacle for patients to receive effective therapy (51), and there is no exception for children with HB (15). This study showed that the most expensive therapy was liver transplantation (99,633.9 CNY), followed by hepatectomy (40,217.5 CNY) and no surgery (8,270.7 CNY). Compared with the real GDP per capita of 70,892 CNY, the per capita disposable income for urban residents of 42,359 $\mathrm{CNY}$, and the per capita disposable income for rural residents of $16,021 \mathrm{CNY}$ in China in 2019 , it posed an economic burden for Chinese families with children suffering from HB. Therefore, it should be given severe considerations when accelerating the survival of children with HB. Further cost-effectiveness analysis on diagnosis and treatment is worth being done (52), which will provide more evidence for the government to invest more on child health and encourage children with $\mathrm{HB}$ to receive more effective therapies.

Although a retrospective analysis is suitable for rare diseases with advantages of time- and cost-saving, there are some biases affecting the results, especially selection bias. To avoid Berkson bias as much as possible, this study includes all children with HB meeting the inclusion and exclusion criteria treated in Renji Hospital from 2013 to 2019. Their survival and death information are tried to be extracted from medical records to avoid McNeyman bias and nonresponse bias. However, this retrospective study has several limitations. First, because of the accessibility of data, some traditional key clinical characteristics, histology, pathology, and staging were not included in this study, including AFP level at diagnosis, histopathology, PRETEXT stage, and metastasis. Second, this is a single-center study with small samples, and the representativeness is limited. Third, it is a short-term follow-up study, and many participants were censored because they are still alive at the last followup. Fourth, considering the economic burden of different surgical therapies, only the average medical expenses were presented and compared rather than a more comprehensive analysis combined with health utilities. Fifth, considering the characteristics of a retrospective study that it is impossible to analyze the causal relationship, the imaging features and pathogenesis of $\mathrm{HB}$ was not explored in this 
study. Therefore, further study, including more factors and participants from various institutions, needs to be designed into a prospective research to verify the results. And a costeffectiveness analysis should be considered in the next step.

\section{Conclusions}

In conclusion, this study showed that surgical therapy could bring a better prognosis, and girls had excellent opportunities for survival. Although some of these study findings have been reported previously, this study could provide some significant evidence nationwide, especially some conflicting factors and from the aspect of economic burden.

\section{Acknowledgments}

Funding: This work was supported by the National Key R\&D Program of China [2017YFC0908100]; and the National Natural Science Foundation of China [81902939].

\section{Footnote}

Reporting Checklist: The authors have completed the STROBE reporting checklist. Available at http://dx. doi. org/10. 21037/gs-20-710

Data Sharing Statement: Available at http://dx. doi. org/10.21037/gs-20-710

Conflicts of Interest: All authors have completed the ICMJE uniform disclosure form (available at http://dx. doi. org/10.21037/gs-20-710). The authors have no conflicts of interest to declare.

Ethical Statement: The authors are accountable for all aspects of the work in ensuring that questions related to the accuracy or integrity of any part of the work are appropriately investigated and resolved. The Ethics Committee approved the study protocol of the Shanghai Jiao Tong University School of Medicine. The written informed consent of all enrolled patients or their parents was obtained. Patient anonymity was guaranteed to be preserved. The study was conducted in accordance with the Declaration of Helsinki (as revised in 2013).

Open Access Statement: This is an Open Access article distributed in accordance with the Creative Commons
Attribution-NonCommercial-NoDerivs 4.0 International License (CC BY-NC-ND 4.0), which permits the noncommercial replication and distribution of the article with the strict proviso that no changes or edits are made and the original work is properly cited (including links to both the formal publication through the relevant DOI and the license). See: https://creativecommons.org/licenses/by-nc-nd/4.0/.

\section{References}

1. Kelly D, Sharif K, Brown RM, et al. Hepatocellular carcinoma in children. Clin Liver Dis 2015;19:433-47.

2. Feng TC, Zai HY, Jiang W, et al. Survival and analysis of prognostic factors for hepatoblastoma: based on SEER database. Ann Transl Med 2019;7:555.

3. Raney B. Hepatoblastoma in children: a review. J Pediatr Hematol Oncol 1997;19:418-22.

4. Herzog CE, Andrassy RJ, Eftekhari F. Childhood cancers: hepatoblastoma. Oncologist 2000;5:445-53.

5. Khanna R, Verma SK. Pediatric hepatocellular carcinoma. World J Gastroenterol 2018;24:3980-99.

6. Darbari A, Sabin KM, Shapiro CN, et al. Epidemiology of primary hepatic malignancies in U.S. children. Hepatology 2003;38:560-6.

7. Mann JR, Kasthuri N, Raafat F, et al. Malignant hepatic tumours in children: incidence, clinical features and aetiology. Paediatr Perinat Epidemiol 1990;4:276-89.

8. Moore SW, Davidson A, Hadley GP, et al. Malignant liver tumors in South African children: a national audit. World J Surg 2008;32:1389-95.

9. Gururangan S, O'Meara A, MacMahon C, et al. Primary hepatic tumours in children: a 26-year review. J Surg Oncol 1992;50:30-6.

10. Pham TH, Iqbal CW, Grams JM, et al. Outcomes of primary liver cancer in children: an appraisal of experience. J Pediatr Surg 2007;42:834-9.

11. Fernandez-Pineda I, Cabello-Laureano R. Differential diagnosis and management of liver tumors in infants. World J Hepatol 2014;6:486-95.

12. Czauderna P, Lopez-Terrada D, Hiyama E, et al. Hepatoblastoma state of the art: pathology, genetics, risk stratification, and chemotherapy. Curr Opin Pediatr 2014;26:19-28.

13. Stiller CA, Pritchard J, Steliarova-Foucher E. Liver cancer in European children: incidence and survival, 1978-1997. Report from the Automated Childhood Cancer Information System project. Eur J Cancer 2006;42:2115-23. 
14. Moore SW, Millar AJ, Hadley GP, et al. Hepatocellular carcinoma and liver tumors in South African children: a case for increased prevalence. Cancer 2004;101:642-9.

15. Yuan XJ, Wang HM, Jiang H, et al. Multidisciplinary effort in treating children with hepatoblastoma in China. Cancer Lett 2016;375:39-46.

16. Perilongo G, Shafford E, Plaschkes J, et al. SIOPEL trials using preoperative chemotherapy in hepatoblastoma. Lancet Oncol 2000;1:94-100.

17. Schnater JM, Aronson DC, Plaschkes J, et al. Surgical view of the treatment of patients with hepatoblastoma: results from the first prospective trial of the International Society of Pediatric Oncology Liver Tumor Study Group. Cancer 2002;94:1111-20.

18. Vinayak R, Cruz RJ Jr, Ranganathan S, et al. Pediatric liver transplantation for hepatocellular cancer and rare liver malignancies: US multicenter and single-center experience (1981-2015). Liver Transpl 2017;23:1577-88.

19. Pham TA, Gallo AM, Concepcion W, et al. Effect of liver transplant on long-term disease-free survival in children with hepatoblastoma and hepatocellular cancer. JAMA Surg 2015;150:1150-8.

20. Wang TY, Han YL, Gao YJ, et al. Retrospective Analysis of Childhood Hepatoblastoma in a Single Centre in China. Clin Oncol (R Coll Radiol) 2019;31:471-8.

21. Qiao GL, Chen Z, Wang C, et al. Pure fetal histology subtype was associated with better prognosis of children with hepatoblastoma: A Chinese population-based study. J Gastroenterol Hepatol 2016;31:621-7.

22. Zhang $\mathrm{Y}$, Zhang $\mathrm{W}$, Tang $\mathrm{S}$, et al. A single-center retrospective study of pediatric hepatoblastoma. Oncol Lett 2016;12:3919-25.

23. Chan KL, Fan ST, Tam PK, et al. Paediatric hepatoblastoma and hepatocellular carcinoma: retrospective study. Hong Kong Med J 2002;8:13-7.

24. Lee CL, Ko YC. Survival and distribution pattern of childhood liver cancer in Taiwan. Eur J Cancer 1998;34:2064-7.

25. Hiyama E. Current therapeutic strategies for childhood hepatic malignant tumors. Int J Clin Oncol 2013;18:943-5.

26. Kremer N, Walther AE, Tiao GM. Management of hepatoblastoma: an update. Curr Opin Pediatr 2014;26:362-9.

27. Horton JD, Lee S, Brown SR, et al. Survival trends in children with hepatoblastoma. Pediatr Surg Int 2009;25:407-12.

28. Meyers RL, Tiao GM, Dunn SP, et al. Liver transplantation in the management of unresectable hepatoblastoma in children. Front Biosci (Elite Ed) 2012;4:1293-302.

29. Evans AE, Land VJ, Newton WA, et al. Combination chemotherapy (vincristine, adriamycin, cyclophosphamide, and 5-fluorouracil) in the treatment of children with malignant hepatoma. Cancer 1982;50:821-6.

30. Barrena S, Hernandez F, Miguel M, et al. High-risk hepatoblastoma: results in a pediatric liver transplantation center. Eur J Pediatr Surg 2011;21:18-20.

31. Liu APY, Ip JJK, Leung AWK, et al. Treatment outcome and pattern of failure in hepatoblastoma treated with a consensus protocol in Hong Kong. Pediatr Blood Cancer 2019;66:e27482.

32. Brown J, Perilongo G, Shafford E, et al. Pretreatment prognostic factors for children with hepatoblastoma-results from the International Society of Paediatric Oncology (SIOP) study SIOPEL 1. Eur J Cancer 2000;36:1418-25.

33. Hishiki T. Current therapeutic strategies for childhood hepatic tumors: surgical and interventional treatments for hepatoblastoma. Int J Clin Oncol 2013;18:962-8.

34. Watanabe K. Current chemotherapeutic approaches for hepatoblastoma. Int J Clin Oncol 2013;18:955-61.

35. Molmenti EP, Wilkinson K, Molmenti H, et al. Treatment of unresectable hepatoblastoma with liver transplantation in the pediatric population. Am J Transplant 2002;2:535-8.

36. Reyes JD, Carr B, Dvorchik I, et al. Liver transplantation and chemotherapy for hepatoblastoma and hepatocellular cancer in childhood and adolescence. J Pediatr 2000;136:795-804.

37. Austin MT, Leys CM, Feurer ID, et al. Liver transplantation for childhood hepatic malignancy: a review of the United Network for Organ Sharing (UNOS) database. J Pediatr Surg 2006;41:182-6.

38. Carceller A, Blanchard H, Champagne J, et al. Surgical resection and chemotherapy improve survival rate for patients with hepatoblastoma. J Pediatr Surg 2001;36:755-9.

39. Ehrlich PF, Greenberg ML, Filler RM. Improved long-term survival with preoperative chemotherapy for hepatoblastoma. J Pediatr Surg 1997;32:999-1002; discussion 1003.

40. King DR, Ortega J, Campbell J, et al. The surgical management of children with incompletely resected hepatic cancer is facilitated by intensive chemotherapy. J Pediatr Surg 1991;26:1074-80; discussion 1080-1.

41. Reynolds M, Douglass EC, Finegold M, et al. Chemotherapy can convert unresectable hepatoblastoma. J 
Pediatr Surg 1992;27:1080-3; discussion 1083-4.

42. Busweiler LA, Wijnen MH, Wilde JC, et al. Surgical treatment of childhood hepatoblastoma in the Netherlands (1990-2013). Pediatr Surg Int 2017;33:23-31.

43. Ezekian B, Mulvihill MS, Schroder PM, et al. Improved contemporary outcomes of liver transplantation for pediatric hepatoblastoma and hepatocellular carcinoma. Pediatr Transplant 2018;22:e13305.

44. Hafberg E, Borinstein SC, Alexopoulos SP. Contemporary management of hepatoblastoma. Curr Opin Organ Transplant 2019;24:113-7.

45. Krstovski N, Janic D, Dokmanovic L, et al. Clinical characteristics and survival of children with hepatoblastoma--single centre experience. Srp Arh Celok Lek 2008;136:603-8.

46. Kaliciński P, Otte JB. Liver transplantation for hepatic malignancies in children--we all need more information. Pediatr Transplant 2009;13:657-8.

47. Buckel E, Silva G, Brahm J, et al. Experience of a single center in liver transplantation in adults and children. Revista Medica De Chile 1996;124:27-36.

Cite this article as: $\mathrm{Yu}$ W, Liu X, Li J, Xi Z, Jin J, Huang H, Ge Y, Xia Q. A single-center retrospective analysis of childhood hepatoblastoma in China. Gland Surg 2020;9(5):1502-1512. doi: 10.21037 /gs-20-710
48. Kirnap M, Ayvazoglu Soy E, Ozcay F, et al. Pediatric liver transplant for hepatoblastoma: a single-center experience. Exp Clin Transplant 2017;15:50-2.

49. Compilation and Examination Expert Group for Guidelines for the diagnosis and treatment of hepatoblastoma (2019). Guidelines for the diagnosis and treatment of hepatoblastoma (2019). J Clin Hepatol 2019;35:2431-4.

50. Perilongo G, Shafford E, Maibach R, et al. Risk-adapted treatment for childhood hepatoblastoma. final report of the second study of the International Society of Paediatric Oncology--SIOPEL 2. Eur J Cancer 2004;40:411-21.

51. Warner EL, Kirchhoff AC, Nam GE, et al. Financial burden of pediatric cancer for patients and their families. J Oncol Pract 2015;11:12-8.

52. Perilongo G, Malogolowkin M, Feusner J. Hepatoblastoma clinical research: lessons learned and future challenges. Pediatr Blood Cancer 2012;59:818-21.

(English Language Editor: J. Chapnick) 